\title{
Unilateral ovarian and fallopian tube agenesis in an infertile patient with a normal uterus
}

\author{
BINGYA CHEN, CHUNBO YANG, ZAYD SAHEBALLY and HANGMEI JIN \\ Department of Gynecology, Women's Hospital, School of Medicine, \\ Zhejiang University, Hangzhou, Zhejiang 310006, P.R. China
}

Received February 6, 2014; Accepted June 13, 2014

DOI: $10.3892 /$ etm.2014.1825

\begin{abstract}
Congenital agenesis of the unilateral adnexa is a condition that has rarely been described in the literature. The current study presents the case of a 26-year-old female who was admitted to the Department of Gynecology at the Women's Hospital of Zhejiang University (Hangzhou, Zhejiang) for primary infertility. The patient was diagnosed with unilateral ovarian and fallopian tubal agenesis, without malformations of the uterus and urinary tract, during diagnostic laparoscopy and hysteroscopy. A literature review was conducted with the aim of determining the possible causes of these anomalies. However, the etiology of the adnexal anomaly remained unclear, although torsion or congenital defects were the most likely explanation. Therefore, the observations of the present study indicate that contralateral tubal pathologies may contribute to sterility.
\end{abstract}

\section{Introduction}

Congenital agenesis of the unilateral adnexa is an uncommon condition that has rarely been described in the literature. In addition, the incidence of adnexal malformations is difficult to determine. It has been suggested to be 1:11,240 (1). Etiologies of ipsilateral ovarian and/or tubal agenesis remain unclear; however, a number of studies have conducted research into this area (1-4). A number of authors suggest that this abnormity is either a congenital malformation or a result of an torsion that occurred to the ovarian pedicle in birth, childhood or adult life $(3,5)$. Adnexal agenesis has always been associated with malformations of the uterus and/or urinary tract. Unilateral absence of the adnexa without a uterine deformity is rarely

Correspondence to: Dr Hangmei Jin, Department of Gynecology, Women's Hospital, School of Medicine, Zhejiang University, 1 Xueshi Road, Hangzhou, Zhejiang 310006, P.R. China

E-mail: hangmei_jinhyd@yeah.net

Key words: unilateral adnexal agenesis, fallopian tube, ovary, congenital anomaly, primary infertility reported. The majority of patients are asymptomatic and can be diagnosed incidentally following a laparoscopy or laparotomy for various gynecological or obstetric complications. The current study reports the case of a 26-year-old female who had been infertile for two years and had been diagnosed with unilateral agenesis of the ovary and fallopian tube during diagnostic laparoscopy and hysteroscopy. In the present study, the literature was reviewed in order to identify possible causes of these anomalies.

\section{Case report}

A 26-year-old nulligravida was admitted to the Department of Gynecology at the Women's Hospital of Zhejiang University (Hangzhou, Zhejiang) with a diagnosis of primary infertility. Informed consent was obtained from the patient for the present study. The patient had not conceived despite regular unprotected intercourse for two years. Menarche occurred at 12 years of age, and the menstrual cycle was regular with 27-28 day intervals and a 5-6-day menstrual period without dysmenorrhea. The overall health of the patient was good and there was no history of abdominopelvic surgery or pelvalgia. On physical examination, no surgical scars were observed. The external genitalia, vagina, cervix and uterus appeared normal on gynecological examination, and the results of sex hormone analysis were also normal. The patient's husband submitted a semen analysis, which was within the normal limits. Transvaginal ultrasonography revealed that the uterus and ovaries (only the right ovary was visualized) were normal. Hysterosalpingography was performed in the Wenling Ruoheng Hospital (Wenling, China) additional local hospital, which revealed a normal uterine cavity; however, the fallopian tubes did not fill bilaterally; the hysterosalpingography was not repeated. Furthermore, genetic analysis revealed a normal karyotype $(46, \mathrm{XX})$.

Subsequently, a diagnostic laparoscopy and hysteroscopy were performed. During the hysteroscopy, the endometrial cavity was observed to be normal, as well as the left and right tubal ostia. The laparoscopy revealed a single, normal-sized uterus with a smooth surface. No adhesion between the uterus and the intestinal serosa, the cecum and the pelvic wall was observed. The rectouterine pouch was inspected and no ectopic tissues were identified. The left adnexa was not completely visualized; however, a 2-cm tubal remnant 
with an intact left round ligament was observed. The right fallopian tube, right ovary (with a corpus luteum) and right round ligament were found to be normal (Fig. 1). The broad ligaments were also normal without any adhesions. In addition, the peritoneal and omental surfaces were analyzed and no ectopic tissues or remnant structures were observed. Methylene blue chromopertubation did not result in spill from the right fallopian tube and the postoperative course was uneventful.

Since adnexal agenesis often coexists with malformations of the urinary tract, abdominopelvic magnetic resonance imaging (MRI) was performed to investigate the urinary system. The MRI scan revealed that the kidneys and ureters were normal bilaterally, while the left ovary was unable to be imaged (Fig. 2).

Unilateral absence of the adnexa is rarely reported without a uterine deformity. Therefore, the present case report prompted a comprehensive literature review. Similar cases reported in the literature, describing unilateral agenesis or the absence of the ovary and fallopian tube without uterine anomalies, are presented in Table I. A total of 25 cases were identified, of which nine cases were diagnosed with primary infertility and seven cases had undergone normal deliveries. In particular, one patient was single, one had contraception by drugs, one had an extrauterine pregnancy and the fertility of the other six cases were not mentioned in the literature. Among the nine nulligravidas, three individuals had obstructed contralateral fallopian tubes, three patients had unobstructed tubes and the condition of the other three were not mentioned in the literature. In certain cases, dysmorphosis of the genital tract coexists with urinary tract anomalies. Two cases were found to be associated with ipsilateral absence of the kidney, while in six cases, urinary tract anomalies were not mentioned.

\section{Discussion}

Congenital absence of the ovary is a rare condition, with one in every 11,240 individuals affected (6); however, the incidence may be higher as it is difficult to estimate the number of cases since the majority are asymptomatic and go unreported. All cases reported in the literature were diagnosed incidentally following a laparoscopy or laparotomy for various gynecological or obstetric complications. The first published case of unilateral ovarian absence was reported in 1923 (7). In recent years, several similar cases have been reported $(1,4,8,9)$, which may be due to the widespread use of laparoscopy for diagnostic purposes; however, the total number of cases remains small.

Adnexal agenesis is often associated with malformations of the uterus and/or urinary tract, including a unicornuate uterus and unilateral renal agenesis (10). Congenital absence of the ovary may be accompanied with total or partial absence of the ipsilateral fallopian tube. However, unilateral absence of the adnexa without a uterine deformity is rarely reported. In the present case report, the uterus was considered normal in shape and structure during diagnostic laparoscopy and hysteroscopy. The literature was reviewed and a number of similar cases without uterine anomalies were identified (Table I). A number of studies have also evaluated the urinary tract in patients with ovarian agenesis $(8,9)$. Among the cases listed, there were two patients with unilateral renal agenesis. In the present case,

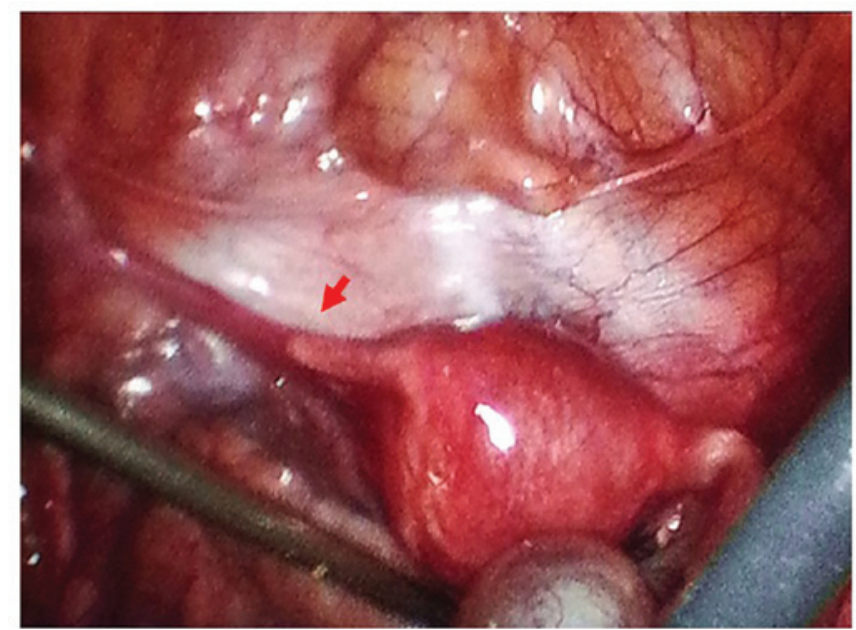

Figure 1. Laparoscopic view reveals a round ligament on the left side and a normal uterus, with no visible left tube or ovary, only a 2-cm tubal remnant. The right fallopian tube and right ovary (with a corpus luteum) were considered to be normal (arrow indicates tubal remnant).

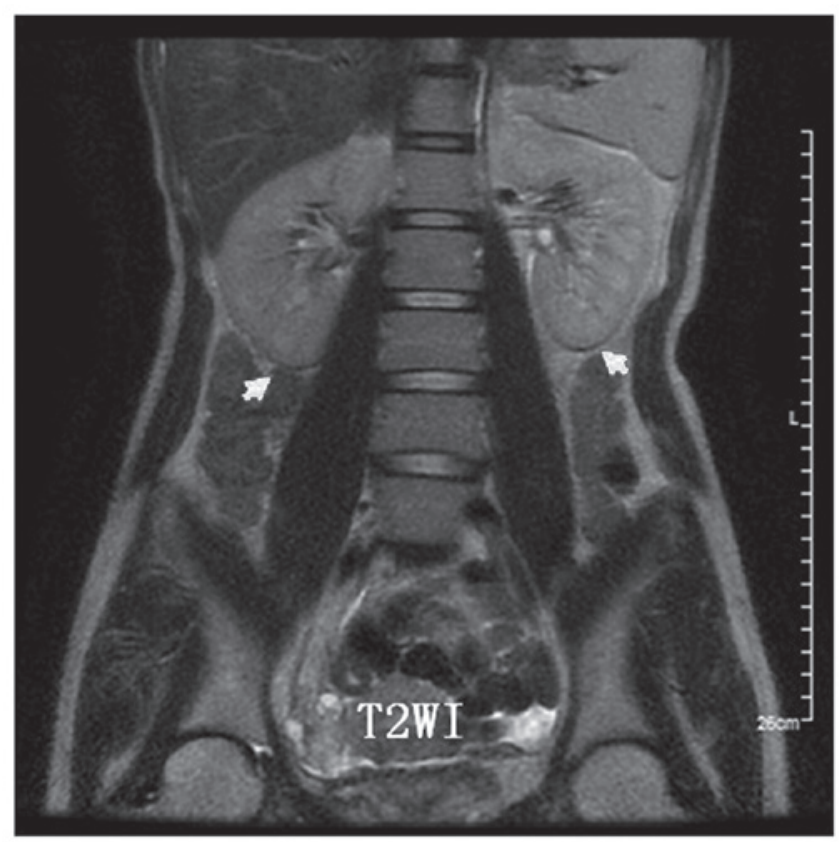

Figure 2. Abdominopelvic magnetic resonance imaging scan showing normal bilateral kidneys (white arrows indicating the normal kidneys).

the results from the MRI scan revealed that the kidneys were normal.

The true etiology of ipsilateral ovarian and/or tubal absence has yet to be elucidated (1-3). The two most likely causes of ipsilateral ovarian and/or tubal absence may include an asymptomatic torsion of the adnexa with consequent organ ischemia and reabsorption $(3,5,11)$, or a defect in the development of the Mullerian and gonadal structures $(3,6,12)$ underlying vascular anomalies (11).

A number of studies have indicated that unilateral absence of the fallopian tube and ovary is the result of adnexal torsion with necrosis and resorption, which may occur antenatally or postnatally $(3,5)$. Furthermore, it has been suggested that symptoms may be minimal or absent, although severe pain in 
Table I. Absence of ovaries and/or fallopian tubes with a normal uterus.

\begin{tabular}{|c|c|c|c|c|}
\hline $\begin{array}{l}\text { First author } \\
\text { (reference) }\end{array}$ & $\begin{array}{l}\text { Ovarian and/or } \\
\text { tubal anomalies }\end{array}$ & $\begin{array}{l}\text { Urinary } \\
\text { anomaly }\end{array}$ & Fertility & $\begin{array}{l}\text { Other notable } \\
\text { observations }\end{array}$ \\
\hline Elkington N (21) & Absent left tube and ovary & No & Normal delivery & \\
\hline Pabuccu E (10) & Absent left tube and ovary & No & Primary infertility & \\
\hline Vaiarelli A (4) & $\begin{array}{l}\text { Complete absence of right } \\
\text { ovary; } 2-\mathrm{cm} \text { proximal } \\
\text { stump of the right tube }\end{array}$ & NM & Primary infertility & $\begin{array}{l}\text { History of acute pelvic } \\
\text { pain } 10 \text { years previously }\end{array}$ \\
\hline Gursoy AY (8) & $\begin{array}{l}\text { Absence of left ovary } \\
\text { and tube }\end{array}$ & $\begin{array}{l}\text { Absence of } \\
\text { left kidney }\end{array}$ & Normal delivery & \\
\hline \multicolumn{5}{|l|}{ Eustace DL (3) } \\
\hline Case 1 & Absent right tube and ovary & No & Primary infertility & \\
\hline Case 2 & Absent right tube and ovary & NM & Normal delivery & \\
\hline \multicolumn{5}{|c|}{ Sivanesaratnam V (6) } \\
\hline Case 1 & Absent left ovary and tube & No & NM & \\
\hline Case 2 & Absent right ovary and tube & No & Primary infertility & Blocked right tube \\
\hline \multicolumn{5}{|l|}{ Mylonas I (5) } \\
\hline Case 1 & Absent right ovary and tube & No & Normal delivery & \\
\hline Case 2 & Absent right ovary and tube & No & Contraception by drugs & \\
\hline Case 3 & Absence right adnexa & No & Primary infertility & Blocked left tube \\
\hline Muppala H (9) & $\begin{array}{l}\text { Absent right ovary, tube and } \\
\text { round ligament }\end{array}$ & $\begin{array}{l}\text { Right renal } \\
\text { agenesis }\end{array}$ & NM & Pyloric stenosis \\
\hline \multicolumn{5}{|l|}{ Uckuyu A (14) } \\
\hline Case 1 & $\begin{array}{l}\text { Absent left distal tubal } \\
\text { segment, streak left ovary }\end{array}$ & No & Primary infertility & Unilateral tubal patency \\
\hline Case 2 & $\begin{array}{l}\text { Absent right distal tubal } \\
\text { segment, normal right ovary }\end{array}$ & No & Primary infertility & Unilateral tubal patency \\
\hline Case 3 & $\begin{array}{l}\text { Twisted left tube, absent } \\
\text { right ovary }\end{array}$ & No & Primary infertility & Unilateral tubal patency \\
\hline Case 4 & Left ovarian agenesis & No & & \\
\hline Tzitzimikas S (22) & $\begin{array}{l}\text { Absence of the left ovary and the } \\
\text { distal part of the ipsilateral tube }\end{array}$ & No & NM & \\
\hline Gotti G (23) & Absent right ovary and tube & No & Extrauterine pregnancy & \\
\hline Rapisarda G (1) & Absent left ovary and tube & NM & Primary infertility & Obstructed right tube \\
\hline Sirisena LA (2) & Absent left ovary and distal tube & No & NM & \\
\hline Georgy FM (13) & Absent left ovary and tube & No & NM & \\
\hline Guan Q (15) & Absent right ovary and tube & NM & Normal delivery & $\begin{array}{l}\text { Teratomas on the } \\
\text { uterine surface }\end{array}$ \\
\hline Liu Q (16) & Absent right ovary and tube & No & NM & $\begin{array}{l}\text { Extraperitoneal huge } \\
\text { serous cystadenoma }\end{array}$ \\
\hline \multicolumn{5}{|l|}{ Ma CL (17) } \\
\hline Case 1 & Absent left ovary & NM & Normal delivery & $\begin{array}{l}\text { Teratomas on the } \\
\text { great omentum }\end{array}$ \\
\hline Case 2 & Absent left ovary and tube & NM & Normal delivery & $\begin{array}{l}\text { Teratomas on the } \\
\text { great omentum }\end{array}$ \\
\hline
\end{tabular}

NM, not mentioned.

the lower abdomen is a typical symptom of adnexal torsion (3). In 1974, Georgy and Viechnicki (13) reported a case with the absence of an ovary and uterine tube. This case supported the torsion hypothesis since a calcific ovary was situated in the Douglas cul-de-sac. The study by Uckuyu et al (14) also supported the torsion hypothesis since separated tubal and 
ovarian tissue remnants were observed in the abdominal cavity. In addition, Vaiarelli et al (4) reported a case where the ovary and ipsilateral fallopian tube were absent. The patient had presented with acute, transient right-sided pelvic pain 10 years previously, which was not diagnosed as adnexal torsion following medical attention. In retrospect, this acute pain may have represented torsion of the right adnexa.

A number of previous cases have demonstrated that unilateral ovarian absence coexists with teratomas on the great omentum or the uterine surface (15-17). Omental cystic teratomas are rare. The authors analyzed the torsion of the ovarian tumors that ruptured and parasitized on the greater omentum (15-17), and it was hypothesized that the incidence may be associated with embryonic developmental abnormalities. While this torsion hypothesis appears plausible, there is no evidence of its occurrence in the present case report. The patient had no history of unexplained abdominal pain, and during laparoscopic surgery, no ectopic tissues or remnant structures on the peritoneal or omental surfaces were observed. However, the absence of symptoms does not exclude the possibility of torsion antenatally.

Developmental abnormalities have been observed in the female reproductive tract, including the fallopian tubes, ovaries, uterus, cervix, vagina and external genitalia. Usually, abnormalities include organs that originate from the Mullerian ducts. In the sixth week of gestation, the bilateral Mullerian ducts migrate towards the midline, meet, form luminal structures, fuse and finally form the uterus and upper one-fifth of the vagina. Rostrally, the Mullerian ducts form fallopian tubes. Any disturbance in the migration, fusion or resorption of these ducts may result in a Mullerian anomaly (18). Paternoster et al (12) presented two cases of absent fallopian tubes, and hypothesized that partial or total unilateral defects of the paramesonephric duct were more common than aplasia of the two ducts. Therefore, a unicornuate uterus, one fallopian tube and one rudimentary or ectopic kidney indicates a defect in the development of all Mullerian structures.

In comparison to Mullerian duct-derived organs, congenital defects of the ovary are rare. Gonadal development depends on accurate germ cell migration, as well as appropriate formation of the urogenital ridge. These processes are regulated by multiple factors and genes (19), and a unilateral defect at any point during this process may prevent ovarian formation. Unilateral ovarian agenesis coexisting with an ipsilateral fallopian tube and a normal uterus is a complicated condition. It has been hypothesized that a defect localized to the region of the genital ridge and the caudal area of the Mullerian duct $(5,20)$ reflects improper development of the urogenital ridge, which affects the development of the fallopian tube in that region. A number of studies have indicated that an inadequate blood supply during the descent into the pelvis of the caudal section of the paramesonephric duct may lead to adnexal agenesis $(3,5,12)$; however, a clear developmental explanation for this malformation has not yet been elucidated. The patient in the current study presented for evaluation with a normal uterus and right adnexa observed during diagnostic laparoscopy and hysteroscopy; thus, excluding the possibility of a unicornuate uterus. Furthermore, the normal karyotype did not support the diagnosis of a chromosomal condition associated with the absence of the fallopian tube and ovary; for example, pure or mixed gonadal dysgenesis (46XY or $45 \mathrm{X} 0 / 46 \mathrm{XY}$ ).

A number of similar patients have been reported in the literature, with anatomic abnormalities observed during evaluations for primary infertility. It is unknown whether unilateral adnexal absence may be a cause of infertility. Several authors have hypothesized that unilateral adnexal absence does not diminish female fertility, particularly when the condition is not accompanied by a uterine malformation (9). Uckuyu et al (14) also investigated the function of the contralateral tube and concluded that unilateral agenesis is a possible factor in patients with infertility. Unilateral absence of the adnexa may reduce the probability of becoming pregnant; however, pregnancy remains possible if there is a functional fallopian tube. Previously, a patient with this condition was reported who had four normal pregnancies that resulted in normal vaginal deliveries (8). The patient presented in the current study was found to have an absent left adnexa and an obstructed right fallopian tube during laparoscopic examination, with no passage of methylene blue solution from the lumen of the right tube; however, the uterus and right ovary were normal. Previous studies have described contralateral occluded tubes $(1,6,5)$. We hypothesized that contralateral tubal pathology may contribute to sterility. However, whether unilateral congenital tubal and ovarian anomalies affect the function of the other tube and the pelvic microenvironment remains unclear.

In conclusion, unilateral ovarian and fallopian tube agenesis is a rare condition. The true etiology of adnexal anomalies remain unclear, although torsion or congenital defects may be the most likely explanations. In addition, the observations of the present study indicate that contralateral tubal pathologies may contribute to infertility.

\section{Acknowledgements}

The study was supported by grants from the Zhejiang Provincial Natural Science Foundation of China and the Subject Fund of Zhejiang Province Department of Education (nos. Y13H040005 and Y201121826, respectively).

\section{References}

1. Rapisarda G, Pappalardo EM, Arancio A and La Greca M: Unilateral ovarian and fallopian tube agenesis. Arch Gynecol Obstet 280: 849-850, 2009.

2. Sirisena LA: Unexplained absence of an ovary and uterine tube. Postgrad Med J 54: 423-424, 1978.

3. Eustace DL: Congenital absence of fallopian tube and ovary. Eur J Obstet Gynecol Reprod Biol 46: 157-159, 1992.

4. Vaiarelli A, Luk J and Patrizio P: Ectopic pregnancy after IVF in a patient with unilateral agenesis of the fallopian tube and ovary and with endometriosis: search of the literature for these associations. J Assist Reprod Genet 29: 901-904, 2012.

5. Mylonas I, Hansch S, Markmann S, Bolz M and Friese K: Unilateral ovarian agenesis: report of three cases and review of literature. Arch Gynecol Obstet 268: 57-60, 2003.

6. Sivanesaratnam V: Unexplained unilateral absence of ovary and fallopian tube. Eur J Obstet Gynecol Reprod Med 22: 103-105, 1986.

7. Alexander HD: True unicornuate uterus and total absence of left broad ligament, round ligament, salpinx, ovary, kidney and ureter. Can Med Assoc J 56: 539, 1947.

8. Gursoy AY, Akdemir N, Hamurcu U and Gozukucuk M: Incidental diagnosis of unilateral renal and adnexal agenesis in a 46-year-old multiparous woman. Am J Case Rep 14: 238-240, 2013. 
9. Muppala H, Sengupta S and Martin JE: Unilateral absence of tube and ovary with renal agenesis and associated pyloric stenosis: communication. Eur J Obstet Gynecol Reprod Biol 137: 123, 2008.

10. Pabuccu E, Kahraman K, Taskın S and Atabekoglu C: Unilateral absence of fallopian tube and ovary in an infertile patient. Fertil Steril 96: e55-e57, 2011.

11. Dahan MH, Burney R and Lathi R: Congenital interruption of the ampullary portion of the fallopian tube. Fertil Steril 85: 18201821,2006

12. Paternoster DM, Costantini W, Uglietti A, Vasile C and Bocconi L: Congenital or torsion-induced absence of fallopian tubes. Two case reports. Minerva Ginecol 50: 191-194, 1998.

13. Georgy FM and Viechnicki MB: Absence of an ovary and uterine tube. Obstet Gynecol 44: 441-442, 1974.

14. Uckuyu A, Ozcimen EE and Sevinc Ciftci FC: Unilateral congenital ovarian and partial tubal absence: report of four cases with review of literature. Fertil Steril 91: 936 e5-e8, 2009.

15. Guan Q and Wu YZ: A full-term pregnancy complicated with uterine mature teratoma and one adnexal absence. Chin J Obstet Gynecol 41: 412, 2006.

16. Liu Q and Sun XB: A retroperitoneal giant serous cystadenoma with unilateral adnexal absence: a case report and review of literature. Chin J Obstet Gynecol 48: 136-137, 2013.
17. Ma CL: Greater omental teratoma with congenital absence of the left ovarian: report of 2 cases. Chin J Clin Obstet Gynecol 7: 302 , 2006.

18. Simpson JL: Genetics of the female reproductive ducts. Am J Med Genet 89: 224-239, 1999.

19. Saitou M, Barton SC and Surani MA: A molecular programme for the specification of germ cell fate in mice. Nature 418: 293-300, 2002.

20. Dare FO, Makinde OO, Makinde ON and Odutayo R: Congenital absence of an ovary in a Nigerian woman. Int $\mathrm{J}$ Gynaecol Obstet 29: 377-378, 1989.

21. Elkington $\mathrm{N}$ and Rahman R: Unexplained unilateral absence of fallopian tube and ovary. The Internet Journal of Gynecology and Obstetrics 11: doi: 10.5580/21a6, 2008.

22. Tzitzimikas S, Fragkos M and Karavida A: Unilateral ovarian absence. Gynecol Surg 10: 93-95, 2013.

23. Gotti G, Ferrone R, Andreoli M, Turini A, Russo P and Di Donato P: Agenesia o assenza annessiale monolaterale? Descrizione di uncaso clinic. It J Gynaecol Obstet 20(2): 87-89, 2008. 